\title{
Evaluation of tuberculosis screening of immigrants in the Netherlands
}

To the Editor:

In European Union/European Economic Area countries, a large proportion of patients with tuberculosis (TB) are born in or are citizens of a country different from the notifying country [1]. Immigrants have an increased risk for $\mathrm{TB}$, depending on the $\mathrm{TB}$ incidence in their country of origin, conditions of their migration and duration of stay in the host country [2]. In low TB-incidence countries, systematic screening of immigrants at high risk for TB pre- or post-entry may be considered a key intervention in the progress towards TB elimination [3, 4]. However, current screening policies and practices in European low-incidence countries differ substantially $[5,6]$.

In the Netherlands, $72 \%$ of the TB cases are foreign born [7]. Since 1966, TB screening of immigrants from non-Western countries is stipulated in the Immigration Act. Immigrants who enter the country for work, study or marriage are referred by the Immigration Department to the Municipal Public Health Services (MPHSs) for screening. Asylum seekers, a specific group of immigrants, are screened in the Netherlands at several reception centres [8]. The main screening method is by chest radiography (CXR). Immigrants younger than 12 years, without evidence of Bacille Calmette-Guérin (BCG) vaccination, are screened with a Tuberculin Skin Test (TST). Individuals with CXR abnormalities suspect for TB undergo further investigations including sputum microscopy and culture. Immigrants from high-incidence countries $(\geqslant 200$ cases per 100000 population) are offered biannual follow-up screening on a voluntary basis for a period of 2 years. Immigrants with CXR abnormalities consistent with inactive TB can be offered either preventive treatment after exclusion of active disease or followed-up radiologically, biannually for 2 years.

The screening policies in the Netherlands are regularly evaluated $[8,9]$. A risk group eligible for screening is defined by a cut-off set at a number needed to screen $\leqslant 2000$, i.e. a prevalence of TB at screening of $\geqslant 50$ cases per 100000 persons screened. In this research letter, we present the results of the evaluation of CXR screening of immigrants (excluding asylum seekers), for the period 2005-2010 and their policy implications. During this period, screening was mandatory for immigrants from all countries except the European Union countries, Australia, Canada, Iceland, Israel, Japan, Liechtenstein, Monaco, New Zealand, Norway, Surinam, Switzerland and the United States of America.

Record-based screening data for the national database "Monitoring for Screening of Immigrants (MSI)" were extracted from the client registration systems used by the TB departments of the MPHSs. The TB patients registered in the MSI database were matched with the Netherlands TB Register (NTR), which is the national TB notification register. Matching was based on date of birth, sex and country of birth. For non-matched patients identified in either the MSI or NTR database, MPHSs were contacted to enable the linkage as previously described [9]. Differences observed between variables for other key information were also verified and corrected where needed. Immigrants were defined to have prevalent TB in case of CXR abnormalities at entry screening and diagnosis of TB within 6 months of entry screening. Results were stratified by age, type of TB, country of origin, WHO-estimated TB incidence in the country of origin and the type of CXR abnormalities.

Over the period of 2005-2010, 117389 immigrants were screened at entry with the largest groups from China (13\%), Turkey (11\%), India (8\%), Morocco (6\%) and Indonesia (5\%). 108 TB cases were detected through entry screening including 100 cases of pulmonary TB (PTB) and eight cases of extrapulmonary TB (ETB) (table 1). TB was confirmed by culture in 84 PTB patients and one ETB patient. Six patients

@ERSpublications

Evaluation of TB screening needed to periodically assess its effectiveness and inform policy decision http://ow.ly/F8zQ30f719D

Cite this article as: van de Berg S, Erkens C, van Rest J, et al. Evaluation of tuberculosis screening of immigrants in the Netherlands. Eur Respir J 2017; 50: 1700977 [https://doi.org/10.1183/13993003.009772017]. 
TABLE 1 Results of chest radiograph screening of immigrants (excluding asylum seekers) in the Netherlands, 2005-2010

\section{Screened $\mathbf{n} \quad$ TB detected $\mathbf{n} \quad$ TB prevalence per} $100000(95 \% \mathrm{Cl})$

\begin{tabular}{lccc}
\hline Age years & & & \\
$0-14$ & 11020 & 3 & $27(5-80)$ \\
$15-34$ & 85352 & 86 & $101(81-124)$ \\
$35-64$ & 20406 & 18 & $88(52-139)$ \\
$\geqslant 65$ & 611 & 1 & $164(2-911)$ \\
Sex & 52866 & 46 & $87(64-116)$ \\
$\quad$ Male & 64135 & 62 & $97(73-121)$ \\
Female & 388 & 0 & \\
Unknown & 31218 & 7 & $22(9-46)$ \\
TB incidence in country of origin per $100000^{\#}$ & 18 & $60(36-94)$ \\
$<50$ & 30019 & 48 & $148(109-196)$ \\
$50-99$ & 32407 & 35 & $161(112-224)$ \\
$100-199$ & 21740 & 0 & $92(75-109)$ \\
$\geqslant 200$ & 2005 & 108 & \\
Unknown & 117389 & & $73(37-132)$ \\
Total & & 11 & $30(8-77)$ \\
Top five immigration countries & 14949 & 4 & $53(17-125)$ \\
$\quad$ China & 13347 & 5 & $80(30-176)$ \\
Turkey & 9310 & 6 & $322(194-504)$ \\
India & 7425 & 19 & \\
Morocco & 5888 & & \\
Indonesia & & & \\
\hline
\end{tabular}

\#: according to the World Health Organization in 2008.

had multi-drug resistant TB. The TB and PTB prevalence of entry screening was 92 (95\% confidence interval [CI]: 75-109) and 85 (CI: 68-102) per 100000, respectively. The TB prevalence of screening was $\geqslant 50$ per 100000 in most subgroups, except in the age group $0-14$ years and in immigrants from countries with a TB incidence $<50$ per 100000 population (table 1).

The TB prevalence at entry screening in our study was lower than in the previous evaluation over the period 1998-2002 (119 per 100000; CI: 93-145) ( $\mathrm{p}=0.08)$ [9]. In particular, the prevalence in immigrants from countries with a TB incidence $<50$ per 100000 population decreased from 54 (CI: 27-96) per 100000 in the period of 1998-2002 to 22 (CI: 9-46) per 100000 in the period 2005-2010 ( $p=0.06$ ), which is lower than the threshold for screening. Based on these results, the Committee for Practical TB Control advised the government to discontinue the mandatory entry screening of immigrants from countries with a TB incidence $<50$ per 100000 population. The Immigration Act was adjusted accordingly and screening of these immigrants was stopped as on January 1, 2015.

Biannual follow-up screening of 16325 immigrants from countries with a TB incidence $\geqslant 200$ per 100000 population with a normal CXR at entry screening identified eight new cases (54 per 100000 screenings; CI: 23-106). Furthermore, 11 out of 507 individuals (2.2\%) with CXR abnormalities suspect for TB at entry screening were diagnosed with TB during the biannual radiological follow-up. Twenty-five individuals with a normal CXR at entry screening who were eligible for follow-up screening were diagnosed with TB (10 PTB and 15 ETB) after presenting with symptoms within 2.5 years after residing in the Netherlands. For eight of the 10 PTB patients, the latest screening had taken place more than 6 months ago. The coverage for the follow-up screening (of immigrants with a normal CXR at entry screening) decreased from $47 \%$ in the first round (after 6 months) to 28\%,21\% and $21 \%$ in the subsequent screening rounds.

A few European low-incidence countries screen immigrants other than asylum seekers systematically and evaluate the results $[10,11]$. A systematic review comparing the yield of entry screening of regular immigrants in several low burden countries found that the yield varied between 101 and 139 per 100000 persons screened, consistent with the findings in our cohort. The prevalence was associated with the TB incidence in the country of origin of the immigrants. In the United Kingdom, the former port-of-entry screening had a lower screening yield (29 per 100000) [12]), while the current pre-entry screening programme found a prevalence of 92 bacteriologically confirmed TB cases per 100000 individuals [5]. 
There are no countries that have reported on follow-up screening of immigrants. Studies have shown that the $\mathrm{TB}$ incidence in immigrant populations remain high several years after residing in the host country $[2,13]$, and that screening for latent tuberculosis infection (LTBI) and providing preventive treatment to those infected, is an effective and potentially cost-saving approach [14]. In the Dutch National Tuberculosis Control Plan 2016-2020 [15], the main strategy aiming to reduce the burden of TB in the Netherlands by $25 \%$ by 2020 , is to supplement or replace radiographic screening by LTBI screening. LTBI screening will be introduced step-wise prioritising children and immigrants from countries with a TB incidence $\geqslant 200$ per 100000 . This approach will be studied in an implementation project supported by ZonMw (TB ENDPoint). The study also includes evaluation of the cost-effectiveness and impact of LTBI screening of the specific subgroups, and these results will guide policy decisions on extending the intervention to other immigrant groups in the Netherlands.

We conclude that periodical evaluation of screening is crucial to assess whether the intervention is effective. In addition, the target groups for screening need regular reassessment and accordingly updated screening policies.

Sarah van de Berg ${ }^{1}$, Connie Erkens ${ }^{1}$, Job van Rest ${ }^{1}$, Susan van den Hof ${ }^{1,2}$, Margreet Kamphorst ${ }^{3}$, Sytze Keizer ${ }^{4}$ and Gerard de Vries ${ }^{1,5}$

${ }^{1}$ KNCV Tuberculosis Foundation, The Hague, The Netherlands. ${ }^{2}$ Dept of Global Health, Academic Medical Center, Amsterdam Institute for Global Health and Development, Amsterdam, The Netherlands. ${ }^{3}$ Dept of Health Care, Custodial Institutions Agency, Ministry of Security and Justice, Utrecht, The Netherlands. ${ }^{4}$ Dept of TB Control, Public Health Service Amsterdam, Amsterdam, The Netherlands. ${ }^{5}$ Centre for Infectious Disease Control, National Institute for Public Health and the Environment (RIVM), Bilthoven, The Netherlands.

Correspondence: Gerard de Vries, KNCV Tuberculosis Foundation, P.O. Box 146, 2501 CC The Hague, The Netherlands. E-mail: gerard.devries@kncvtbc.org

Received: May 122017 | Accepted after revision: July 112017

Conflict of interest: None declared.

\section{References}

1 World Health Organization. Tuberculosis surveillance and monitoring in Europe 2016. Geneva, World Health Organization, 2016.

2 Vos AM, Meima A, Verver S, et al. High incidence of pulmonary tuberculosis persists a decade after immigration, the Netherlands. Emerg Infect Dis 2004; 10: 736-739.

3 Lönnroth K, Migliori GB, Abubakar I, et al. Towards tuberculosis elimination: an action framework for low-incidence countries. Eur Respir J 2015; 45: 928-952.

4 Aldridge RW, Zenner D, White PJ, et al. Prevalence of and risk factors for active tuberculosis in migrants screened before entry to the UK: a population-based cross-sectional study. Lancet Infect Dis 2016; 16: 962-970.

5 Coker R, Bell A, Pitman R, et al. Tuberculosis screening in migrants in selected European countries shows wide disparities. Eur Respir J 2006; 27: 801-807.

6 Dara M, Solovic I, Sotgiu G, et al. Tuberculosis care among refugees arriving in Europe: a ERS/WHO Europe Region survey of current practices. Eur Respir J 2016; 48: 808-817.

7 Slump E, Erkens CGM, van Hunen R, et al. Tuberculose in Nederland 2015. Surveillance rapport [Tuberculosis in the Netherland 2015. Surveillance Report]. Bilthoven, National Institute for Public Health and the Environment (RIVM), 2016.

8 De Vries G, Van Rest J, Meijer W, et al. Low yield of screening asylum seekers from countries with a tuberculosis incidence of $<50$ per 100000 population. Eur Respir J 2016; 47: 1870-1872.

9 Erkens C, Slump E, Kamphorst M, et al. Coverage and yield of entry and follow-up screening for tuberculosis among new immigrants. Eur Respir J 2008; 32: 153-161.

10 Klinkenberg E, Manissero D, Semenza JC, et al. Migrant tuberculosis screening in the EU/EEA: yield, coverage and limitations. Eur Respir J 2009; 34: 1180-1189.

11 Arshad S, Bavan L, Gajari K, et al. Active screening at entry for tuberculosis among new immigrants: a systematic review and meta-analysis. Eur Respir J 2010; 35: 1336-1345.

12 Severi E, Maguire $\mathrm{H}$, Ihekweazu C, et al. Outcomes analysis of new entrant screening for active tuberculosis in Heathrow and Gatwick airports, United Kingdom 2009/2010. BMC Infect Dis 2016; 16: 178.

13 Aldridge RW, Zenner D, White PJ, et al. Tuberculosis in migrants moving from high-incidence to low-incidence countries: a population-based cohort study of 519955 migrants screened before entry to England, Wales, and Northern Ireland. Lancet 2016; 388: 2510-2518.

14 Pareek M, Watson JP, Ormerod LP, et al. Screening of immigrants in the UK for imported latent tuberculosis: a multicentre cohort study and cost-effectiveness analysis. Lancet Infect Dis 2011; 11: 435-444.

15 de Vries G, Riesmeijer R. National Tuberculosis Control Plan 2016-2020 : towards elimination. www.rivm.nl/en/ Documents_and_publications/Scientific/Reports/2016/maart/National_Tuberculosis_Control_Plan_2016_2020_ Towards_elimination Date last accessed: September 18 2017. Date last updated: March 242016. 